\title{
Octopus (Enterocopus dofleini) Liver Extract Displays Triglyceride-Lowering Effect in HepG2 Cells
}

\author{
Yasushi Hasegawa*, Tomohiko Hori \\ Email address: \\ hasegawa@mmm.muroran-it.ac.jp (Y. Hasegawa) \\ ${ }^{*}$ Corresponding author
}

Department of Applied Sciences, Muroran Institute of Technology, Hokkaido, Japan

To cite this article:

Yasushi Hasegawa, Tomohiko Hori. Octopus (Enterocopus dofleini) Liver Extract Displays Triglyceride-Lowering Effect in HepG2 Cells. Agriculture, Forestry and Fisheries. Vol. 6, No. 6, 2017, pp. 214-218. doi: 10.11648/j.aff.20170606.15

Received: October 11, 2017; Accepted: October 26, 2017; Published: November 22, 2017

\begin{abstract}
Fatty liver disease is characterized by the accumulation of triglycerides and other fats in the liver cells and is believed to be a risk of later chronic liver disease. Diet is one of the key ways to treat fatty liver disease. Octopus (Enterocopus dofleini) liver is eaten in some regions in Japan, but mostly discarded. For utilization of octopus liver, the lipidlowering effect of octopus (Enterocopus dofleini) liver extract was investigated using human hepatoma cells (HepG2 cells). The present study showed that the octopus liver extract reduced the triglyceride content, but not cholesterol content, in HepG2 cells. Treatment with the octopus liver extract increased the mRNA expression of genes for peroxisome proliferator-activated receptor (PPAR)- $\alpha$, medium chain acyl-CoA dehydrogenase (MCAD), and acyl-CoA oxidase (ACO) associated with $\beta$ oxidation. On the contrary, the extract did not change the mRNA expression of genes for sterol regulatory element-binding protein (SREBP)-1, acetyl-CoA carboxylase (ACC), fatty acid synthase (FAS), stearoyl-CoA desaturase (SCD)-1, and peroxisome proliferator-activated receptor (PPAR)- $\gamma$ involved in fatty acid synthesis. These results suggest that octopus liver extract may decrease the triglyceride level in HepG2 cells by promoting $\beta$-oxidation, suggestive of its usefulness as a food for lowering triglycerides in liver.
\end{abstract}

Keywords: Efficient Utilization, Octopus Liver Extract, HepG2 Cells, Triglyceride

\section{Introduction}

Liver plays a central role in the biogenesis of major metabolites such as glucose, fatty acids, and cholesterol. Excessive alcohol intake causes fatty liver under a condition characterized by large accumulation of triglycerides and other fats in liver cells [1-3]. The accumulation of fatty acid in the liver depends on the balance between the processes of its delivery and removal and may be associated with a decrease in the mitochondrial fatty acid $\beta$-oxidation, increase in the endogenous fatty acid synthesis, or enhancement in the delivery of fatty acids to the liver [4]. Fatty liver disease exhibits a gradual progression from simple hepatic steatosis to steatohepatitis. Diet is one of the key ways to treat fatty liver disease. Dietary intake of fishes such as salmon, sardines, and trout, which are rich sources of omega- 3 fatty acids, is known to protect the liver against fatty liver and hepatic steatohepatitis $[5,6]$. Caffeine intake is associated with a lower risk of fatty liver disease, suggestive of the potential protective role of caffeine [7]. Consumption of green tea rich in high-density catechins is shown to decrease liver fat content and inflammation in fatty liver disease [8].

Octopus arms and body parts are common ingredients in Japanese cuisine. However, the consumption of octopus liver is rare and hence, liver is mostly discarded. The present study investigated the biological activity of octopus liver extract for the efficient utilization and reports its triglyceride-lowering effect in liver using human hepatoma HepG2 cells.

\section{Materials and Methods}

\subsection{Preparation of Octopus Liver Extract}

Enterocopus dofleini was collected in Hokkaido and the liver tissues isolated. Liver tissues $(200 \mathrm{~g})$ were homogenized in deionized water $(500 \mathrm{~mL})$ and centrifuged at $12,000 \times g$ for $15 \mathrm{~min}$. The supernatant was collected and used as the 
octopus extract.

\subsection{Cell Culture}

Human hepatoma HepG2 cells were purchased from RIKEN Cell Bank (Tsukuba, Japan). Cells were maintained in modified Eagle's medium (MEM) supplemented with 10\% fetal calf serum (FCS) under $95 \%$ air and $5 \% \mathrm{CO}_{2}$.

\subsection{Cell Viability Assay of HepG2 Cells}

HepG2 cells were seeded in a 96-well plate at a density of $4 \times$ $10^{3}$ cells/well in $100 \mu \mathrm{L}$ of medium. After $24 \mathrm{~h}$, octopus extract was added to the culture medium at various concentrations. Following treatment with the octopus extract for $96 \mathrm{~h}$, the cell number was quantified using the 3-(4,5-dimethylthiazol-2-yl)2,5-diphenyltetrazolium bromide (MTT) assay [9]. Briefly, MTT $(0.5 \mathrm{mg} / \mathrm{mL}$ in phosphate-buffered saline [PBS]) was added to each well and incubated for $4 \mathrm{~h}$ at $37^{\circ} \mathrm{C}$. The medium was carefully aspirated and $200 \mu \mathrm{L}$ of $20 \%$ sodium dodecyl sulfate (SDS) was added to solubilize the colored product. After $24 \mathrm{~h}$, the absorbance was recorded at $570 \mathrm{~nm}$ wavelength using a scanning multi well spectrophotometer.

\subsection{Cytotoxicity}

Cytotoxicity was assessed by measuring the lactate dehydrogenase (LDH) activity. HepG2 cells were treated with the octopus extract for $96 \mathrm{~h}$. Following treatment, the culture medium $(50 \mu \mathrm{L})$ was transferred to a 96-well culture plate and the cytotoxicity was measured using an LDH cytotoxicity test kit (Wako).

\subsection{Triglyceride and Cholesterol Content in HepG2 Cells}

HepG2 cells were seeded in a 96-well plate at a density of $1 \times 10^{5}$ cells/well in $100 \mu \mathrm{L}$ of medium, followed by treatment with the octopus extract at the indicated concentration. After $96 \mathrm{~h}$, the medium was removed and cells washed twice with PBS. Triglycerides and cholesterol were extracted with hexane: propanol (3:2) solution [10] and the extract dried. Triglyceride and cholesterol contents were measured using the Triglyceride $\mathrm{E}$ test and Cholesterol $\mathrm{E}$ test commercial kit (Wako, Osaka, Japan).

\subsection{Oil red-O Dye Staining}

Intracellular lipid accumulation was evaluated with the oil red- $O$ dye staining. HepG2 cells were washed with PBS and fixed in $3.7 \%$ formaldehyde solution containing PBS at $4{ }^{\circ} \mathrm{C}$ for $5 \mathrm{~min}$. The fixed cells were washed twice with PBS and stained with $0.16 \%$ oil red- $O$ solution for $20 \mathrm{~min}$. After removing the oil red- $O$ solution, cells were washed thrice with distilled water. The cell-incorporated dye was extracted with $60 \%$ isopropanol and the absorbance of the extract measured at $490 \mathrm{~nm}$ wavelength.

\subsection{Semi-quantitative Reverse Transcription Polymerase Chain Reaction (RT-PCR) Analysis}

Total RNA was extracted from HepG2 cells incubated in the absence or presence of octopus extract using an RNAiso Plus (Takara, Shiga, Japan), as per the manufacturer's protocol. First-strand complementary DNA (cDNA) was synthesized from $20 \mu \mathrm{g}$ of total RNA using oligo (dT) primer and PCR was carried out using $0.05 \mu \mathrm{g}$ of cDNA template. The specific forward and reverse primers used were as follows: $\beta$-actin, 5'-TTGTTACAGGAAGTCCCTTGCC-3' (forward) and 5'-ATGCTATCACCTCCCCTGTGTG-3' (reverse); peroxisome proliferator-activated receptor (PPAR)$\gamma$ 5'-ACTTATCCTGTGGTCCCCGG-3' (forward) and 5'CCGACAGAAAGGCACTTGTGA-3' (reverse); PPAR- $\alpha$ 5'TCTTCACGATGCTGTCCTCCT $-3^{\prime}$ (forward) and $5^{\prime}$ CTATGTTTAGAAGGCCAGGC $-3^{\prime}$ (reverse); medium chain acyl-CoA dehydrogenase (MCAD), 5'CTACCAAGTATGCCCTGGAAAG-3' (forward) and 5'TGTGTTCACGGGCTACAATAAG-3' (reverse); acyl-CoA oxidase (ACO), 5'-GGGCATGGCTATTCTCATTGC-3' (forward) and 5'CGAACAAGGTCAACAGAAGTTAGGTTC-3' (reverse); sterol regulatory element-binding protein (SREBP)-1, 5'GGAGGGGTAGGGCCAACGGCCT-3' (forward) and 5'CATGTCTTCGAAAGTGCAATCC-3' (reverse); acetyl-CoA carboxylase (ACC), 5'GAAGGGCTTATATTGCCTATGACCTTAAC-3' (forward) and 5'-GGGCAGCATGAACTGGAATT-3' (reverse); fatty acid synthase (FAS), 5'-ACAGGGACAACCTGTAGTTCT3' (forward) and 5'-CTGTGGTCCCACTTGATGAGT-3' (reverse); and stearoyl-CoA desaturase (SCD)-1, 5'CCTCTACTTGGAAGACGACATTCGC-3' (forward) and 5'-GCAGCCGAGCTTTGTAAGAGCGGT-3' (reverse). The intensity of the amplified bands was estimated using ImageJ software. The mRNA expression levels were normalized to actin mRNA level. The amplification cycles were determined based on the relationship between the amount of PCR product detected and cycle number.

\subsection{Statistical Analysis}

Each experiment was performed in duplicates or triplicates. Data were combined from at least four repeats and expressed as the mean \pm standard deviation (SD). Data were analyzed using Student's t-test.

\section{Results and Discussion}

\subsection{Effects of the Octopus Extract on Viability of HepG2 Cells}

The effects of the octopus extract on the viability of HepG2 cells was investigated. Following treatment of HepG2 cells with the octopus extract, the number of viable cells was determined using the MTT assay (Figure 1). The octopus extract did not inhibit cell proliferation and changed cell viability. In addition, investigation of the cytotoxicity of the octopus extract using LDH activity revealed that the extract did not induce any toxicity at a concentration of $0.1 \mathrm{mg} / \mathrm{mL}$. The octopus extract was used at concentrations of 0.02 and $0.1 \mathrm{mg} / \mathrm{mL}$ in the following experiments. 
(a) MTT assay

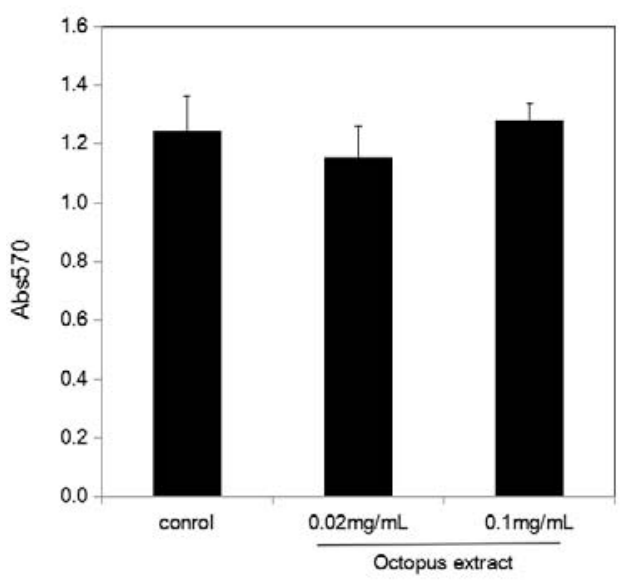

(b) LDH activity

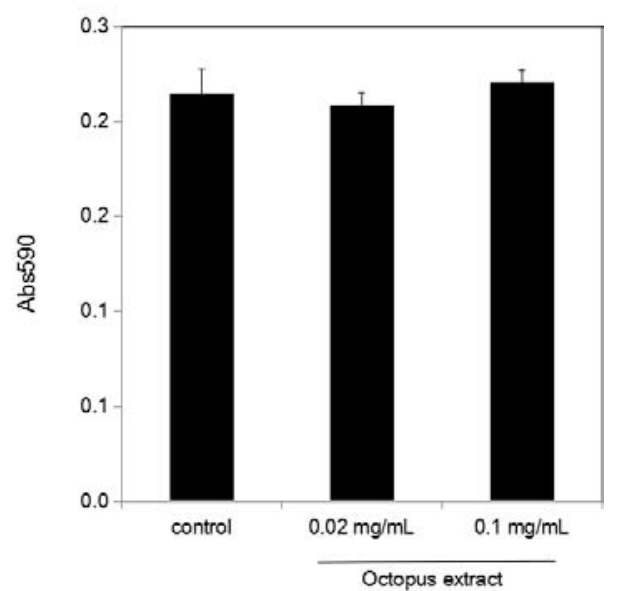

Figure 1. Toxicity of octopus extract against HepG2 cells. HepG2 cells were treated with the octopus extract for 96 h at the indicated concentration. Cells were cultured in the absence or presence of octopus extract. Data represent results obtained from six wells of a 96-well plate, and the bars represent the means \pm standard deviations $(S D)$.

\subsection{Effect of the Octopus Extract on Triglyceride and Cholesterol Accumulation in HepG2 Cells}

We investigated the triglyceride and cholesterol content in HepG2 cells in the presence or absence of the octopus extract and found that the extract decreased triglyceride accumulation by about $70 \%$ at $0.1 \mathrm{mg} / \mathrm{mL}$ concentration as compared with the control (Figure 2a). No change in the cholesterol content was observed in the presence of the extract (Figure 2b). This result was also confirmed by
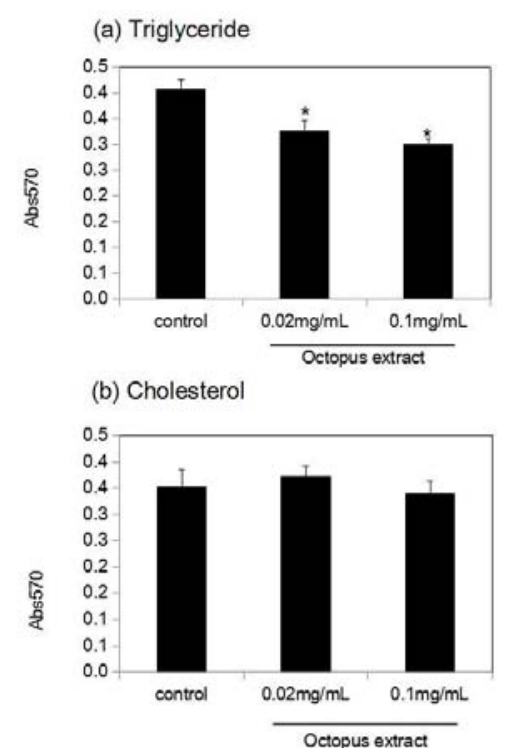

estimating the intracellular lipid accumulation using oil red$O$ dye staining. In presence of the octopus extract, a decrease in the lipid droplet in HepG2 cells was observed as compared with the control. These results suggest that octopus extract may decrease the triglyceride content in HepG2 cells. Next the underlying mechanism of action was investigated by measuring the mRNA expression of genes associated with fatty acid synthesis and $\beta$-oxidation.
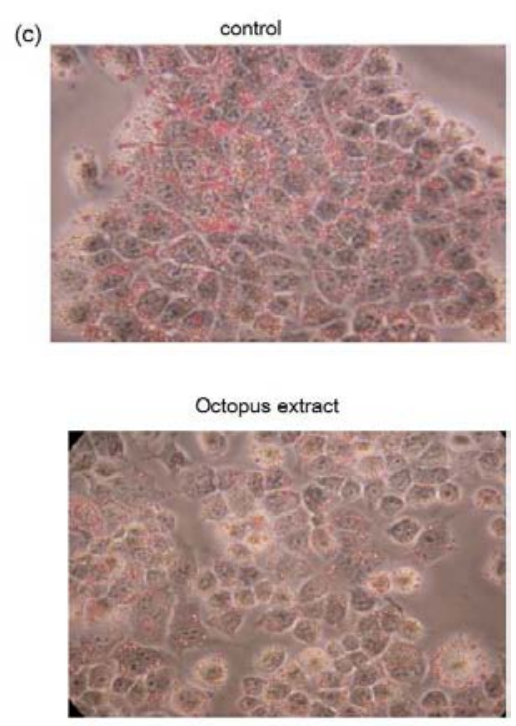

Figure 2. Effect of octopus extract on triglyceride (a) and cholesterol (b) contents in HepG2 cells. HepG2 cells were treated with the octopus extract at the indicated concentration. After $96 \mathrm{~h}$, triglyceride and cholesterol were extracted and their levels measured. Data represent results obtained from six wells of a 96-well plate, and the bars represent the means \pm SD. Statistical significance was determined by Student's t-test: *p $<0.05$ relative to control. (c) Lipid droplet in Hep 2 cells were stained with the oil red-O dye and photographed under phase-contrast microscopy.

\section{3. mRNA Expression of Genes Associated with Fatty Acid Synthesis and $\beta$-oxidation}

Lipid accumulation in liver is caused by an increase in the free fatty acid uptake of liver, impaired fatty acid $\beta$ oxidation, or increased incidence of de novo lipogenesis. We investigated the mRNA expression of genes associated with $\beta$-oxidation. Studies suggest that PPAR- $\alpha$, a member of the 
nuclear receptor superfamily of transcription factors, is involved in the regulation of fatty acid $\beta$-oxidation [11-13]. Activation of PPAR- $\alpha$ induces an increase in the mRNA expression of its target genes such as ACO and MCAD, thereby resulting in the upregulation of $\beta$-oxidation. Treatment of HepG2 cells with the octopus extract resulted in a significant increase in the mRNA expression of PPAR- $\alpha$, MCAD, and ACO (Figure 3). In particular, a two-fold increase in the expression of ACO was observed following treatment with the octopus extract. Next, we investigated the mRNA expression of genes associated with fatty acid synthesis. SREBP-1 and PPAR- $\gamma$ affect the transcription of genes such as ACC, FAS, and SCD1, which regulate the de novo lipid synthesis $[14,15]$. Treatment with the octopus extract did not change the expression of SREBP-1, PPAR- $\gamma$, ACC, FAS, and SCD (Figure 4), suggesting that the octopus extract has no effect on the lipid synthesis but promotes $\beta$ oxidation activity in HepG2 cells. Lipid content is regulated by a balance between fatty acid synthesis and uptake and $\beta$ oxidation. The triglyceride-lowering effect of the octopus extract may be attributed to its ability to upregulate the expression of genes associated with $\beta$-oxidation. Triglyceride accumulation has been linked to an increased risk of fatty liver and associated diseases. Octopus liver may be effective as a food for the prevention of fatty liver. Future studies should estimate the in vivo triglyceride-lowering effect of octopus extract in the liver.

Polyunsaturated fatty acids, polyphenol, and 1-carnitine are known to upregulate the expression of a rate-limiting enzyme in the $\beta$-oxidation process in HepG 2 cells, leading to a decrease in the plasma lipid concentration [16-18]. Taurine and fish oil decreased the white adipose tissue weight in type 2 diabetic mice through the inhibition of fatty acid synthesis and promotion of $\beta$-oxidation in the liver [19]. The $\beta$-oxidation stimulatory effect of the octopus extract may be effective in decreasing the fat weight and lowering plasma lipid concentration.
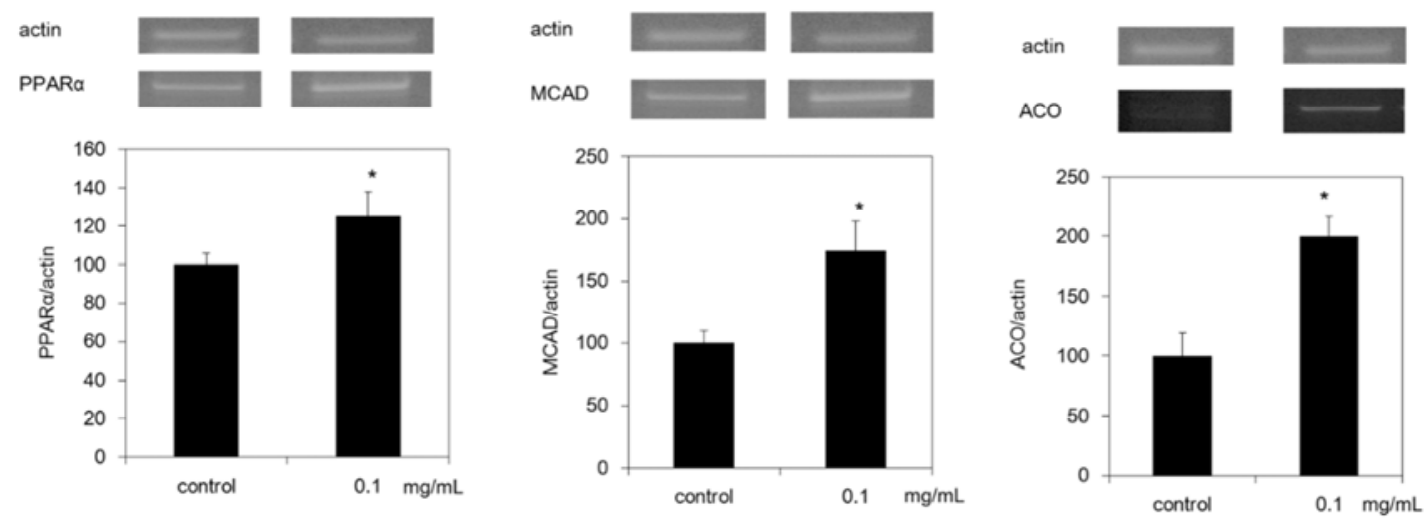

Figure 3. Semi-quantitative RT-PCR analysis of genes associated with $\beta$-oxidation. After treatment of HepG2 cells with the octopus extract at the indicated concentration, total RNA was extracted and semi-quantitative RT-PCR performed. The bars represent the means $\pm S D$. Statistical significance was determined by Student's t-test: *p $<0.05$ relative to control.
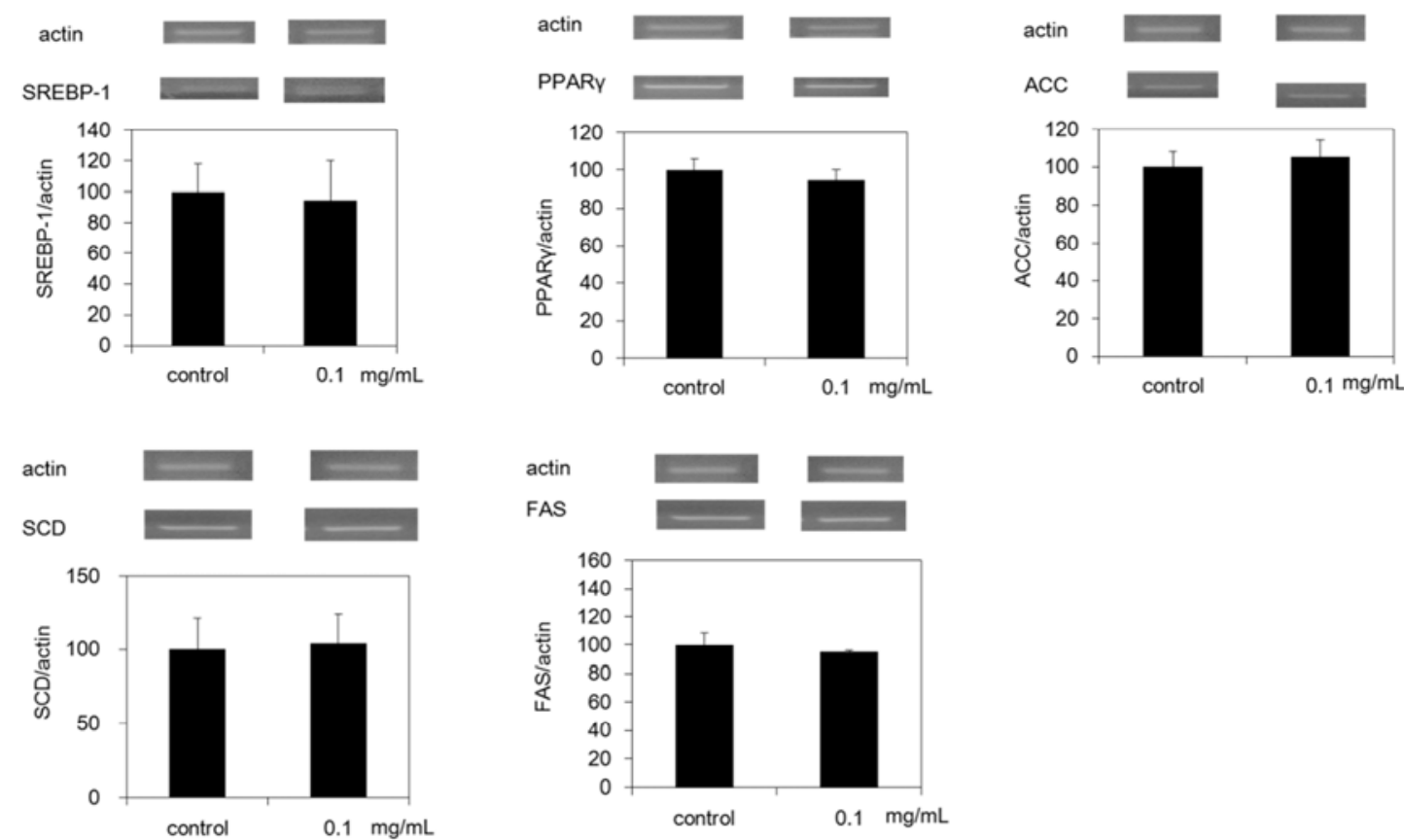

Figure 4. Semi-quantitative RT-PCR analysis of genes associated with fatty acid synthesis. After treatment of HepG2 cells with the octopus extract at the indicated concentration, total RNA was extracted and semi-quantitative RT-PCR performed. The bars represent the means $\pm S D$. 


\section{Conclusion}

Results presented here suggest that octopus liver extract may decrease the triglyceride level in HepG2 cells by promoting $\beta$-oxidation, suggesting that octopus liver may be effective as a food for the prevention of fatty liver.

\section{References}

[1] Lewis JR, Mohanty SR. Nonalcoholic fatty liver disease: a review and update. Digestive Diseases and Sciences, 55, 2010, 560-578.

[2] Reddy JK, Sambasiva Rao M. Lipid Metabolism and Liver Inflammation. II. Fatty liver disease and fatty acid oxidation. American Journal of Physiology Gastrointestinal and Liver Physiology, 290, 2006, G852-G858.

[3] Benedict M, Zhang X. Non-alcoholic fatty liver disease: An expanded review. World Journal of Hepatology, 9, 2017, 715732.

[4] Mantena, SK, King AL, Andringa KK, Eccleston HB, Bailey SM. Mitochondrial dysfunction and oxidative stress in the pathogenesis of alcohol- and obesity-induced fatty liver diseases. Free Radical Biology \& Medicine, 44, 2008, 12591272 .

[5] Parker HM, Johnson NA, Burdon CA, Cohn JS, O'Connor HT, George J. Omega-3 supplementation and non-alcoholic fatty liver disease: A systematic review and meta-analysis. Journal of Hepatology, 56, 2012, 944-951.

[6] Jump DB, Lytle KA, Depner CM, Tripathy S. Omega-3 polyunsaturated fatty acids as a treatment strategy for nonalcoholic fatty liver disease. Pharmacology and Therapeatics, 16, 2017, in press.

[7] Birerdinc A, Stepanova M, Pawloski L, Younossi ZM. Caffeine is protective in patients with non-alcoholic fatty liver disease. Alimentary Pharmacology \& Terapeutics 35, 2011, 76-82.

[8] Sakata R, Nakamura T, Torimura T, Ueno T, Sata M. Green tea with high-density catechins improves liver function and fat infiltration in non-alcoholic fatty liver disease (NAFLD) patients: A double-blind placebo-controlled study. International Journal of Molecular Medicine 32, 2013, 989994.

[9] Manthorpe M, Fagnani R, Skaper SD, Varon S. An automated colorimetric microassay for neuronotrophic factors. Brain Research, 390, 1986, 191-198.

[10] Granot E, Schwiegelshohn B, Tabas I, Gorecki M, Vogel T, Carpentier YA, Deckelbaum RJ. Effects of particle size on cell uptake of model triglyceride-rich particles with and without apoprotein E. Biochemistry, 33, 1994, 15190-15197.
[11] Kim SM, Lee B, An HJ, Kim DH, Park KC, Noh SG, Chung KW, Lee EK, Kim KM, Kim DH, Kim SJ, Chun P, Lee HJ, Moon HR, Chung HY Novel PPAR $\alpha$ agonist MHY553 alleviates hepatic steatosis by increasing fatty acid oxidation and decreasing inflammation during aging. Oncotarget, 8, 2017, 46273-46285.

[12] Crabb DW, Galli A, Fischer M, You M. Molecular mechanisms of alcoholic fatty liver: role of peroxisome proliferator-activated receptor alpha. Alcohol, 34, 2004, 3538 .

[13] Felix AD, Takahashi N, Takahashi M, Katsumata-Tsuboi R, Satoh R, Soon Hui T, Miyajima K, Nakae D, Inoue H, Uehara M. Extracts of black and brown rice powders improve hepatic lipid accumulation via the activation of PPAR in obese and diabetic model mice. Bioscience, Biotechnology, and Biochemistry, 22, 2017, 1-3.

[14] Feng X, Yu W, Li X, Zhou F, Zhang W Shen Q, Li J, Zhang C, Shen P. Apigenin, a modulator of PPAR $\gamma$, attenuates HFDinduced NAFLD by regulating hepatocyte lipid metabolism and oxidative stress via Nrf2 activation. Biochemical Pharmacology, 136, 2017, 136-149.

[15] Wang LF, Wang XN, Huang CC, Hu L, Xiao YF, Guan XH, Qian YS, Deng KY, Xin HB. Inhibition of NAMPT aggravates high fat diet-induced hepatic steatosis in mice through regulating Sirt1/AMPK $\alpha /$ SREBP1 signaling pathway. Lipids in Health \& Disease, 16, 2017, 82.

[16] Radler U, Stangl H, Lechner S, Lienbacher G, Krepp R, Zeller E, Brachinger M, Eller-Berndl D, Fischer A, Anzur C, Schoerg G, Mascher D, Laschan C, Anderwald C, Lohninger A. A combination of ( $\omega-3)$ polyunsaturated fatty acids, polyphenols and L-carnitine reduces the plasma lipid levels and increases the expression of genes involved in fatty acid oxidation in human peripheral blood mononuclear cells and HepG2 cells. Annals of Nutrition and Metabolism, 58, 2011, 133-140.

[17] Shimoda H, Tanaka J., Kikuchi M., Fukuda T., Ito H, Hatano T, Yoshida T. Effect of polyphenol-rich extract from walnut on diet-induced hypertriglyceridemia in mice via enhancement of fatty acid oxidation in the liver. Journal of Agricultural and Food Chemistry, 57, 2009, 1786-1792.

[18] Wang S, Huang Y, Xu H, Zhu Q, Lu H, Zhang M, Hao S, FangC, Zhang D, Wu X, Wang X, Sheng J. Oxidized tea polyphenols prevents lipid accumulation in liver and visceral white adipose tissue in rats. European Journal of Nutrition, 56, 2017, 2037-2048.

[19] Mikami N, Hosokawa M, Miyashita K. Dietary combination of fish oil and taurine decreases fat accumulation and ameliorates blood glucose levels in type 2 diabetic/obese KKA(y) mice. Journal of Food Science, 77, 2012, H114-H120. 